\title{
Estimation of Salivary Matrix Metalloproteinases- 12 (MMP- 12) Levels Among Patients Presenting With Oral Submucous Fibrosis and Oral Squamous Cell Carcinoma
}

\section{Zohra Saleem ( $\nabla$ zohra_mymani@hotmail.co.uk)}

Dow University of Health Sciences

Abdul Hafeez Shaikh

Dow University of Health Sciences

Uzma Zaman

Dow University of Health Sciences

Shaheen Ahmed

Dow University of Health Sciences

Muhammad Mansoor Majeed

Altamash institute of dental medicine

Anum Kazmi

Dow University of Health Sciences

Waqas Ahmed Farooqui

Dow University of Health Sciences

\section{Research Article}

Keywords: Matrix metalloproteinase, MMP-12, Oral Squamous cell carcinoma, Oral Submucous fibrosis, Salivary biomarker, ELISA

Posted Date: March 3rd, 2021

DOl: https://doi.org/10.21203/rs.3.rs-254722/v1

License: (c) (i) This work is licensed under a Creative Commons Attribution 4.0 International License. Read Full License

Version of Record: A version of this preprint was published at BMC Oral Health on April 23rd, 2021. See the published version at https://doi.org/10.1186/s12903-021-01571-7. 


\section{Abstract}

\section{Background:}

Oral squamous cell carcinoma is a global threat and accounts for approximately $90 \%$ of malignant oral lesions. The emergence of oral carcinoma is linked to precancerous lesions which act as precursors of the disease. Matrix metalloproteinases appear to play a significant role in the pathogenesis of both precancerous conditions and oral malignancies due to their participation in the remodelling of the extracellular matrix.

\section{Methodology:}

This is an analytical study conducted at Dow University of Health Sciences, Karachi, Pakistan. Unstimulated saliva samples were collected from healthy, oral submucous fibrosis and oral squamous cell carcinoma patients. The level of MMP-12 was estimated using enzyme-linked immunosorbent assay (ELISA). One-way analysis of variance (ANOVA) was run to determine if MMP-12 levels differ between cases and controls which was preceded by posthoc Tuckey test.

\section{Results}

A significant difference in salivary MMP-12 expression was observed in OSF and OSCC. The expression of salivary MMP-12 was higher in cases compared to controls. The mean MMP-12 expression in OSCC was found higher than in OSF cases.

\section{Conclusion}

MMP-12 expression increases as the healthy patient advances to OSF and OSCC. The study results also demonstrate higher MMP-12 expression in OSCC patients as compared to OSF. Therefore, estimation of salivary MMP-12 serves as a useful non-invasive early diagnostic tool in the diagnosis of oral submucous fibrosis and oral squamous cell carcinoma.

\section{Introduction:}

Oral cancer poses a major health threat all over the world and holds the highest mortality rate among all malignancies worldwide [1]. Despite an increase in the knowledge on prevention and treatment of the disease, an increasing number of cases every year is quite evident. Oral cavity cancers appear to be among the most prevalent cancers globally and occur in nearly one-fifth of all cancers in males and onetenth of all cancers in females globally [2].

The emergence of oral carcinoma is linked to precancerous lesions which act as precursors of the disease [3]. These precancerous lesions include erythroplakia, leukoplakia and oral submucous fibrosis (OSF) with oral submucous fibrous having high potential for malignant transformation among people

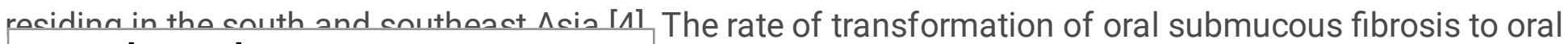
Loading [MathJax]/jax/output/CommonHTML/jax.js 
squamous cell carcinoma (OSCC) is roughly around $2.3-7.6 \%$ [5]. The risk is increased in patients consuming heavy tobacco and alcohol [6]. Early and correct diagnosis of highly suspicious precancerous lesions helps in timely treatment and prevents transformation into malignancy. Tumour responds well to treatment modalities in the early stage as compared to the late stage. This is evident by the outcome that approximately $80 \%$ of patients have an expected 5 - years of survival [7].

Histological examination is the gold standard but, to some extent, not practicable because of the nature and therapeutic response of the tumour [8]. It has a major drawback of being an invasive method as well as painful and time-consuming $[9,10]$. Therefore, the focus is being directed towards non-invasive methods for the detection of oral squamous cell carcinoma. Changes in human genetics can be identified in patients body fluid like saliva, cerebrospinal fluid, blood serum and urine. These body fluids reflect an alteration in proteins and nucleic acid and therefore can be considered effective biomarkers for detection of oral squamous cell carcinoma at an early stage [11].

Until now, several oral salivary biomarkers have been studied but not enough data is available for a biomarker that could aid in primary OSCC detection at the non-detectable stage or precursor stage [12]. Identifying a biomarker may serve threefold benefits; detection of the tumour at an early stage, serving as a prognostic marker and a therapeutic target [12]. Matrix metalloproteinase (MMPs) are considered one of those potential biomarkers that can theoretically fulfil all these purposes. It has been suggested that estimation of MMPs levels in various tissue fluids serve as an accessible, non-time-consuming and noninvasive tool for diagnosis of primary disease along with subsequent prognostic monitoring [12].

Several studies observed MMPs expression in oral cancer and demonstrated enhanced expression of different MMPs and advancement of the disease as compared to controls. Generally, increased expression of MMP-1, MMP-2, MMP-3, MMP-7, MMP-9, MMP-13 and MMP-14 is associated with cancer advancement which relates to poor cell differentiation, tumour invasion, distant metastasis and poor prognosis [12]. The protein concentrations of MMP-1, MMP-2, MMP-3 and MMP-9 also have been seen to be greater in OSCC tumour tissue in contrast to control tissue [13].

The aim and objective of this study is to estimate and compare the levels of salivary MMP-12 in patients presenting with oral submucous fibrosis and patients presenting with oral squamous cell carcinoma.

\section{Methodology:}

It is a descriptive \& analytical cross-sectional study. The study was conducted on patients presenting to centres of Dow University of health sciences, Karachi. There were three groups of patients contributing to the study. Each group consisted of an equal number of participants (30 participants) based on inclusion and exclusion criteria. Group 1 represented healthy individuals.

Group 2 consisted of patients presenting with oral submucous fibrosis and group 3 represented patients presenting to the OPD with biopsy-proven oral squamous cell carcinoma. 
The questionnaire for OSF and OSCC patients is a department- designed (OMFS department, Dow University of Health Sciences). It has been pretested on several patients for the past few years for maintaining the record, hence it's a validated instrument. The first part of the questionnaire contained questions related to socio-demographic data. The second part of the questionnaire contained questions about disease status.

The Performa for healthy participants was filled out to facilitate whether the participant qualifies the inclusion criteria.

The healthy participants included students, OPD assistants, friends and family members.

The questionnaire consists of five parts which include patient's demographic features and personal information, medical history, tobacco and alcohol habits, oral hygiene practice and other information related to oral dryness [14].

Informed consent in accordance with Helsinki's declaration was sought from all participants and assurance of confidentiality about personal data was provided.

\section{Sample collection:}

For saliva sample collection, patients were seated in a clean and comfortable environment with the dental chair in an upright position. They were advised not to communicate during the procedure and not to forcefully spit or cough up mucus but let the saliva drool in a $15 \mathrm{~mL}$ Falcon tubes once it has been collected on the floor of the mouth. Around $2-5 \mathrm{~mL}$ of unstimulated saliva sample was gathered in a falcon tube by the passive drooling method which is considered as an assuring alternative for minimizing potential sources of error [14]. The effect of possible environmental factors (tobacco chewing, betel nut chewing, smokeless tobacco consumption, smoking) was controlled at the analysis phase through Analysis of covariance (ANCOVA).

The supernatant obtained was carefully transferred equally into 3-4. Eppendorf tubes (microcentrifuge tubes- $1 \mathrm{~mL})$ via pipette Juster $(1000 \mu)$. After disinfection and labelling with the patient's name and hospital registration number, microcentrifuge tubes were stored in the freezer at $-80^{\circ} \mathrm{C}$ until further ELISA investigation. ELISA was performed according to the manufacturer's instruction manual and the coloured product was read immediately at 450nm wavelength. Excess sample was washed from the plate. Each sample was analysed in duplicate for statistical analysis. MMP12 concentration in saliva was calculated with the help of standard curve using a known concentration of standard MMP12.

Readings were obtained by using ELISA reader software. Data were analysed using SPSS 23.0 version (SPSS, Inc., Chicago, IL). Pearson correlation test was applied to analyse the correlation between MMP-12 level and age. Comparison of means among genders was tested by applying independent sample $t$-test. The statistical analysis was run with a significance level of $p<0.05$. One-way analysis of variance was run to determine if MMP-12 levels differ between cases and controls which was preceded by post hoc 


\section{Results:}

The sociodemographic data of all the subjects included in the study are presented in Table 1. This data includes ages, gender, marital status, ethnicities, occupation and oral habits of all participants.

Table 1: Association of Sociodemographic data of cases and controls

\begin{tabular}{|c|c|c|c|}
\hline Category & $\begin{array}{l}\text { CONTROLS } \\
\mathrm{n}(\%)\end{array}$ & $\begin{array}{l}\text { OSF } \\
\mathrm{n}(\%)\end{array}$ & $\begin{array}{l}\text { OScC } \\
\mathrm{n}(\%)\end{array}$ \\
\hline $\begin{array}{l}\text { Age } \\
\text { (Mean S.D) }\end{array}$ & 28.876 .81 & 33.2712 .43 & 47.1313 .38 \\
\hline Gender & $5(16.6 \%)$ & $25(83.3 \%)$ & $20(66.6 \%)$ \\
\hline $\begin{array}{l}\text { Male } \\
\text { Female }\end{array}$ & 25 (83.3\%) & $5(16.6 \%)$ & $10(33.3 \%)$ \\
\hline Marital status & $8(26.6 \%)$ & $10(33.3 \%)$ & $1(3.3 \%)$ \\
\hline $\begin{array}{l}\text { Unmarried } \\
\text { Married }\end{array}$ & $22(73.3 \%)$ & $20(66.6 \%)$ & $29(96.6 \%)$ \\
\hline Ethnicity & $10(33.3 \%)$ & $3(10 \%)$ & $8(26.6 \%)$ \\
\hline Sindhi & $8(26.6 \%)$ & $17(56.6 \%)$ & $14(46.6 \%)$ \\
\hline $\begin{array}{l}\text { Urdu speaking } \\
\text { Other }\end{array}$ & $12(40 \%)$ & $10(33.3 \%)$ & $8(26.6 \%)$ \\
\hline Occupation & $23(76.6 \%)$ & 19 (63.3\%) & $20(66.6 \%)$ \\
\hline $\begin{array}{l}\text { Employed } \\
\text { Unemployed }\end{array}$ & $7(23.3 \%)$ & $11(36.6 \%)$ & $10(33.3 \%)$ \\
\hline Oral habits & $7(23.3 \%)$ & $17(56.6 \%)$ & $10(33.3 \%)$ \\
\hline Betel nut & $4(13.3 \%)$ & $6(20 \%)$ & 7 (23.3\%) \\
\hline Tobacco smoking & $0(0 \%)$ & $7(23.3 \%)$ & $4(13.3 \%)$ \\
\hline $\begin{array}{l}\text { Smokeless tobacco } \\
\text { None }\end{array}$ & $19(63.3 \%)$ & $0(0 \%)$ & $9(30 \%)$ \\
\hline Total & 30 & 30 & 30 \\
\hline
\end{tabular}


cases and controls. A positive moderate correlation was observed between age and MMP-12 expression among cases and controls $(r=0.35, p=0.001)$. It demonstrates that as age increases, MMP-12 levels also increase.

A test was run to compare mean MMP-12 expression between gender among cases and controls. A highly statistically significant mean difference in MMP-12 expression was observed between genders $(\mathrm{p}<$ 0.001). Higher mean MMP-12 expression was found in Males $(M=12.5 \mathrm{ng} / \mathrm{ml}$ ) compared to Females ( $M$ $=5.59 \mathrm{ng} / \mathrm{ml})$. (Table 2).

Table 2: Mean comparison of Matrix metalloproteinases- 12 expressions with genders

\begin{tabular}{|llll|}
\hline Gender & $\mathbf{n}$ & Mean (S.D) & M.D (p-value $\left.{ }^{\text {ó}}\right)$ \\
\cline { 1 - 3 } Male & 50 & $12.5(5.84)$ & \multirow{2}{*}{$6.91(<0.001)^{\star *}$} \\
Female & 40 & $5.59(6.72)$ & \\
\cline { 1 - 3 } Total & 90 & & \\
\hline
\end{tabular}

ó- p-value computed using Independent t- test

*-Significant at 0.05

M.D- Mean difference

Oral habits of cases and controls were recorded. A statistical test was applied to compare the means of MMP-12 levels among participants with various oral habits. A statistically significant difference in means was observed $(p<0.05)$ among participants with distinct oral habits (Table 3$)$.

Table 3: Descriptive ANOVA- MMP-12 expression \& oral habits

\begin{tabular}{|llll|}
\hline Category & $\mathbf{n}$ & Mean (SD) & MSE (p-value ${ }^{\circ}$ ) \\
\cline { 1 - 3 } Betel nut & 34 & $10.5(5.8)$ & \multirow{2}{*}{ 44.46 $\left(0.003^{*}\right)$} \\
\cline { 1 - 3 } Tobacco smoking & 17 & $10.5(6.9)$ & \\
\cline { 1 - 3 } Smokeless tobacco & 11 & $13.8(4.2)$ & \\
\cline { 1 - 3 } None & 28 & $5.6(8.0)$ & \\
Total & 90 & & \\
\hline
\end{tabular}

Ò - p-value computed using One-way ANOVA

*-Significant at 0.05 
MSE- Mean square error

(Table 4) demonstrates a significant difference in mean between smokeless tobacco consumers and those with non-significant oral habits. MMP-12 levels among smokeless tobacco consumers appear higher as compared to participants with non-significant oral habits. A statistically significant difference in the mean is also evident in betel nut consumers and participants with non-significant oral habits. Higher MMP-12 levels appear in betel nut consumers as compared to individuals with no significant oral habits.

Table 4: Mean comparison of MMP-12 expression among individuals with distinct oral habits

\begin{tabular}{|lll|}
\hline Comparison & Mean difference & P-value $^{\text {oे }}$ \\
\hline Smokeless tobacco vs betel nut consumers & 3.3 & 0.48 \\
\hline Smokeless tobacco vs tobacco smoking & 3.3 & 0.56 \\
\hline Smokeless tobacco vs non-significant oral habit & 8.2 & $0.005^{\star}$ \\
\hline Betel nut vs tobacco smoking & 0.04 & $>0.99$ \\
\hline Betel nut vs non-significant oral habits & 4.8 & $0.026^{*}$ \\
\hline Tobacco smoking vs non-significant oral habits & 4.8 & 0.091 \\
\hline
\end{tabular}

ò-p-value computed using Post hoc Tuckey test

*- Significant at 0.05

The difference in mean MMP-12 level among cases and controls was analysed. The result demonstrated a statistically significant difference in salivary MMP-12 means among cases and controls $(p<0.001)$. A pvalue of $<0.025$ was considered significant (Table 5 ).

Table 5: Descriptive ANOVA- MMP-12 expression ( $\mathrm{ng} / \mathrm{ml}$ ) among cases \& controls

\begin{tabular}{|c|c|c|c|}
\hline Category & $\mathbf{n}$ & Mean (SD) & MSE (p-value ò) \\
\hline Controls & 30 & $0.82(0.45)$ & \multirow[t]{4}{*}{$12.48\left(<0.001^{\star}\right)$} \\
\hline OSF & 30 & $12.53(3.2)$ & \\
\hline OSCC & 30 & $14.92(5.1)$ & \\
\hline Total & 90 & $9.43(7.1)$ & \\
\hline
\end{tabular}

Ò- p-value computed using One-way ANOVA

MSE- Mean square error 
The control group demonstrated significantly different salivary MMP12 expression compared to cases belonging to Oral Submucous Fibrosis $(p<0.001)$ \& Oral Squamous Cell Carcinoma $(p<0.001)$ group respectively. Table 6 depicts the mean comparison of MMP-12 expression among the control, OSF and OSCC groups at statistically significant mean level $(p=0.014)$.

The unilateral hypothesis was applied for evaluating whether cases mean is greater than control mean and whether OSCC mean is greater than OSF mean. Cases groups demonstrated higher MMP-12 expression as compared to controls and OSCC group showed higher levels in comparison with the OSF group, therefore, the order of mean is OSCC $>0 S F>$ Controls.

Table 6: Mean comparison of MMP-12 expression ( $\mathrm{ng} / \mathrm{ml}$ ) among cases \& controls

\begin{tabular}{|lll|}
\hline Pairs & Mean difference & P-value $^{\text {ò }}$ \\
\hline OSF vs controls & 11.70 & $<0.001^{\star}$ \\
\hline OSCC vs controls & 14.09 & $<0.001^{\star}$ \\
\hline OSCC vs OSF & 2.39 & $0.014^{\star}$ \\
\hline
\end{tabular}

Ò- p-value computed using Post hoc Tuckey test

*- Significant at 0.025

\section{Discussion:}

Level of MMP-12 appears to be highly expressed in a wide range of cancers, including colorectal, gastric, skin nasopharyngeal and lung cancer [15-20]. A study was carried out to identify expression of LIFR, PIK3R1 and MMP- 12 in gall bladder carcinoma. This study validated MMP-12 as a significant prognostic biomarker in this rare and aggressive tumour [21]. Another study was conducted to analyse the expression of MMP-12 level in patients presenting with laryngeal squamous cell carcinoma. There was a poorer degree of tumour differentiation as the expression of MMP-12 went higher [22]. Elevated levels of MMP-12 were also correlated with pathological stage and metastasis of lung adenocarcinoma. Hence, targeting MMP-12 for the treatment of lung adenocarcinoma seemed promising. In addition to this, high expression of MMP-12 was observed in oesophageal squamous cell carcinoma compared to normal epithelial cell [23]. Due to its functional properties and its role in tissue destructive disease, MMP 12 can be used as a biomarker for various oral diseases [24]. It plays a significant part in tumorigenesis and progression. This includes tumour growth, migration, invasion and tumour metastasis $[12,25,26]$. MMP12 is recommended as a diagnostic biomarker for OSCC due to its significant sensitivity and specificity [12].

The current study consisted of participants with a minimum age of 18 years and maximum age of 78 vears with the mean ade of 36.4213 .60 vears. The mean age between OSF, OSCC and control groups Loading [MathJax]/jax/output/CommonHTML/jax.js 
was found to be significant. Statistical analysis was applied to observe the relationship between salivary MMP-12 expression and age of participants including both cases and controls group. The results demonstrated a statistically significant direct relationship between the two variables which explains that as the age increases, MMP-12 expression in saliva increases. In contrast to our findings, lower salivary MMP-12 levels were reported in a study in individuals aged 40-60 years as compared to individuals under 40 years [24].

A higher proportion (56\%) of research participants were males whereas $44 \%$ were females. A statistically significant difference was observed in salivary MMP-12 expression among genders, implying higher mean expression of salivary MMP-12 in males as compared to females. However, no difference in salivary MMP-12 was observed between males and females in a study focusing on MMP-12 levels in patients with periodontal inflammation [24].

The study also illustrates the oral habits of participants. Majority of the participants were betel nut consumers followed by patients reporting tobacco smoking as their oral habit. A comparison was made between distinct oral habits and salivary MMP-12 expression. A significant difference was observed between the MMP-12 expression of individuals with distinct oral habits. Smokeless tobacco consumers demonstrated higher MMP-12 expression compared to other oral habits. However, individuals who reported tobacco and betel nut as their oral habit demonstrated lower MMP-12 expression comparatively. In contrast to the results mentioned, another study evidences a non-significant difference in MMP-12 expression among patients with different oral habits, including smoking, alcohol and betel nut chewing [27].

The results of the current study demonstrated a statistically significant difference in salivary MMP-12 expression in OSF and OSCC group as compared to healthy participants. Cases groups (OSF and OSCC) showed higher salivary MMP-12 expression and lower expression was observed in the control group. The results coincide with a previous study which indicated a high association of salivary protease spectrum with oral health status [28]. It demonstrated increased proteases levels in OSCC patients as compared to patients presenting with other oral diseases. MMP- 12 was detected only in the saliva of patients with OSCC along with other MMPs, such as MMP-1, MMP-2, MMP-3, MMP-10 and MMP-13. In addition to this, MMP-1, MMP-2, MMP-10 and MMP-12 were also observed to be significantly increased in patients with OSCC in comparison to healthy patients and patients with oral benign masses (OBM) and mild chronic periodontitis (CPD). The concentration of salivary MMP-12 in OSCC patients demonstrated in this study is around $1300 \mathrm{pg} \cdot \mathrm{ml}^{-1}$ which is comparatively more than healthy $\left(700 \mathrm{pg} \cdot \mathrm{ml}^{-1}\right)$, oral benign mass (900 pg. $\left.\mathrm{ml}^{-1}\right)$ and chronic periodontal disease patients (900 pg. $\left.\mathrm{ml}^{-1}\right)$ [28].

A cohort study carried out in Sweden on 436 participants aimed to investigate salivary MMP-12 levels about various aspects of oral health. The influence of non-disease covariates on MMP-12 levels was also assessed. The results revealed an association between MMP-12 levels and percentage of gingival pockets $4 \mathrm{~mm}$. The study concluded that MMP-12 reflect the various aspect of periodontal disease and 
the levels are contrarily affected by the presence of tumour [24]. The results of this study also corroborate the results of our study in which MMP-12 levels are affected in the presence of the tumor.

A study was conducted to compare the MMP12 level in patients presenting with OSCC and verrucous carcinoma (VC) in tissue samples. The study results showed that VCs were devoid of epithelial MMP-12 expressions compared to SCC [29]. Another study was undertaken to estimate serum MMPs levels in OSCC patients compared to healthy participants. Serum level of MMP-12 was notably arisen in OSCC patients as compared to healthy participants [12].

MMP-12 expression found elevated in patients with chronic periodontitis with identification of CD68+ CD14+CD64+ cells [24]. Also, the expression of MMP 12 in tissue sample goes high in patients with extracapsular spread compared to those without extracapsular spread[20]. Hence, it may be a useful predictive marker for extracapsular spread (ECS) in head and neck tumours[20].

In recent years, the prevalence of OSF has increased from $8.3 / 10^{5}$ to $16.2 / 10^{5}[30,31]$. The rate of malignant transformation to oral cancer is $9.13 \%$ and there's 29.26 times higher risk in OSF patients as compared to non-OSF patients [32,33]. In Pakistan, OSF is contemplated as a public health concern as oral malignancies are one of the most common malignancies reported [34]. Also, oral habits like tobacco smoking and consumption of betel nut and smokeless tobacco are major risk factors of OSF and are fairly common in Pakistan. Studying the role of several markers present in saliva will help in devising a non-invasive investigation for OSF diagnosis, which will ultimately result in early diagnosis of OSF and prevent it from advancing to OSCC if treated promptly. Since OSF is an oral potentially malignant disorder and is fairly common in our part of the world, we've studied the expression of MMP-12 in OSF patients along with OSCC patients.

Statistically, a significant difference in salivary MMP-12 level was observed in OSF patients in comparison with controls. OSF patients demonstrated higher MMP-12 levels as compared to controls. However, in comparison with salivary MMP-12 in OSCC, salivary MMP-12 in OSF demonstrated lower expression explaining the increase in MMP-12 expression as an oral potentially malignant disorder (OSF) drifts towards malignancy (OSCC). Since the significant difference in salivary MMP-12 levels is observed between OSF and OSCC, this investigation may serve as a useful non-invasive device for differentiating OSCC from specifically last stage OSF in which patients present with nil mouth opening and the surgeon suspects a lesion intraorally.

\section{Conclusion:}

The current study indicates salivary MMP-12 expression in patients presenting with oral submucous fibrosis and biopsy-proven oral squamous cell carcinoma. We observed a statistically significant difference in salivary MMP-12 expression in cases as compared to controls with higher expression in OSF and OSCC patients. The study results also demonstrate higher expression of salivary MMP-12 in OSCC as 
compared to OSF. Therefore, estimation of salivary MMP-12 may serve as a useful non-invasive early diagnostic tool in the diagnosis of oral submucous fibrosis and oral squamous cell carcinoma.

\section{Abbreviations}

OSCC- Oral squamous cell carcinoma

OSF- Oral submucous fibrosis

MMP- Matrix metalloproteinases

ELISA: enzyme-linked immunosorbent assay

ANOVA: One-way analysis of variance

OBM: Oral benign mass

CPD- Mild chronic periodontitis

VC: Verrucous carcinoma

\section{Declarations}

\section{Ethical approval \& consent to participate:}

The study was approved by Dow University of health sciences' Institutional Review Board (IRB1322/DUHS/Approval/2019/71). Informed consent to participate was taken from all participants according to Helsinki's declaration.

\section{Consent for publication:}

The consent for publication was taken from all participants.

\section{Availability of data and materials:}

The datasets used and analysed during the current study are available from principal investigator (corresponding author) on reasonable request.

\section{Competing interests:}

The authors declare that they have no competing interests.

\section{Funding:}

The current study did not receive any funding. 
Author's contribution:

ZS: Study concept, data collection, Sample processing, drafting

AS: Data collection, Analysis interpretation

UZ: Sample processing (Lab work), Final approval

SA: Literature search, critical evaluation

MM: Sample processing, Data entry

AK: Literature search, drafting

WA: Data analysis, Analysis interpretation

Acknowledgements:

$\mathrm{N} / \mathrm{A}$

\section{References}

1. Ahmad T, Singh S: Cancer and Precancerous Lesions of Oral Cavity; Our Experience. JIDA: Journal of Indian Dental Association 2016, 10(7).

2. Saleem Z, Abbas SA, Nadeem F, Majeed MM: The habits and reasons of delayed presentation of patients with oral cancer at a tertiary care hospital of a third world country Pakistan Journal of Public Health 2018, 8(3):165-169.

3. Goyal D, Goyal P, Singh H, Verma C: An update on precancerous lesions of oral cavity. Int J Med Dent Sci 2013, 2:70-75.

4. Ekanayaka RP, Tilakaratne WM: Oral submucous fibrosis: review on mechanisms of malignant transformation. Oral surgery, oral medicine, oral pathology and oral radiology 2016, 122(2):192-199.

5. Selvam NP, Dayanand AA: Lycopene in the management of oral submucous fibrosis. Asian J Pharm Clin Res 2013, 6(3):58-61.

6. Lingen MW, Abt E, Agrawal N, Chaturvedi AK, Cohen E, D'Souza G, Gurenlian J, Kalmar JR, Kerr AR, Lambert PM: Evidence-based clinical practice guideline for the evaluation of potentially malignant disorders in the oral cavity: a report of the American Dental Association. The Journal of the American Dental Association 2017, 148(10):712-727. e710.

7. Wang Z, Jiang L, Huang C, Li Z, Chen L, Gou L, Chen P, Tong A, Tang M, Gao F: Comparative proteomics approach to screening of potential diagnostic and therapeutic targets for oral squamous cell carcinoma. Molecular \& Cellular Proteomics 2008, 7(9):1639-1650.

8. Khan MSR, Siddika F, Xu S, Liu XL, Shuang M, Liang HF: Diagnosing oral squamous cell carcinoma 
9. Ishikawa S, Sugimoto M, Kitabatake K, Sugano A, Nakamura M, Kaneko M, Ota S, Hiwatari K, Enomoto A, Soga T: Identification of salivary metabolomic biomarkers for oral cancer screening. Scientific reports $2016,6: 31520$.

10. Kaur J, Jacobs R, Huang Y, Salvo N, Politis C: Salivary biomarkers for oral cancer and pre-cancer screening: a review. Clinical oral investigations 2018, 22(2):633-640.

11. Javaid MA, Ahmed AS, Durand R, Tran SD: Saliva as a diagnostic tool for oral and systemic diseases. Journal of oral biology and craniofacial research 2016, 6(1):67-76.

12. Choudhry N, Sarmad S, Gondal AJ: Estimation of serum matrix metalloproteinases among patients of oral squamous cell carcinoma. Pakistan Journal of Medical Sciences 2019, 35(1):252.

13. Baker E, Leaper D, Hayter J, Dickenson A: The matrix metalloproteinase system in oral squamous cell carcinoma. British Journal of Oral and Maxillofacial Surgery 2006, 44(6):482-486.

14. Bhattarai KR, Kim H-R, Chae H-J: Compliance with Saliva Collection Protocol in Healthy Volunteers: Strategies for Managing Risk and Errors. International Journal of Medical Sciences 2018, 15(8):823831.

15. Zheng J, Chu D, Wang D, Zhu Y, Zhang X, Ji G, Zhao H, Wu G, Du J, Zhao Q: Matrix metalloproteinase12 is associated with overall survival in Chinese patients with gastric cancer. Journal of surgical oncology 2013, 107(7):746-751.

16. Kahlert C, Pecqueux M, Halama N, Dienemann H, Muley T, Pfannschmidt J, Lasitschka F, Klupp F, Schmidt T, Rahbari N: Tumour-site-dependent expression profile of angiogenic factors in tumourassociated stroma of primary colorectal cancer and metastases. British journal of cancer 2014, $110(2): 441$.

17. Chung I-C, Chen L-C, Chung A-K, Chao M, Huang H-Y, Hsueh C, Tsang N-M, Chang K-P, Liang Y, Li H-P: Matrix metalloproteinase 12 is induced by heterogeneous nuclear ribonucleoprotein $\mathrm{K}$ and promotes migration and invasion in nasopharyngeal carcinoma. BMC cancer 2014, 14(1):348.

18. Lv F, Wang J, Wu Y, Chen H, Shen X: Knockdown of MMP12 inhibits the growth and invasion of lung adenocarcinoma cells. International journal of immunopathology and pharmacology 2015, 28(1):7784.

19. Liu L, Sun J, Li G, Gu B, Wang X, Chi H, Guo F: Association between MMP-12-82AVG polymorphism and cancer risk: a meta-analysis. International journal of clinical and experimental medicine 2015, 8(8):11896.

20. Kim JM, Kim HJ, Koo BS, Rha KS, Yoon Y-H: Expression of matrix metalloproteinase-12 is correlated with extracapsular spread of tumor from nodes with metastasis in head and neck squamous cell carcinoma. European Archives of Oto-Rhino-Laryngology 2013, 270(3):1137-1142.

21. Li MD, Zhao XY, Xu MX, Cai Z, Yuan WJ, Cui WY: Identification of LIFR, PIK3R1, and MMP12 as novel prognostic signatures in gallbladder cancer using network-based module analysis. Frontiers in oncology $2019,9: 325$.

22. El-Hennawi DE-DM, El Tabbakh MT, Sabek NAE-A, Abou-halawa AS, Fareed AM: High Mobility Group Loading [MathJax]/jax/output/CommonHTML/jax.js ase 12 (MMP12) Genes Expression in Egyptian Patients with 
Laryngeal Squamous Cell Carcinoma. Egyptian Journal of Ear, Nose, Throat and Allied Sciences 2019, 20(2):94-98.

23. Han F, Zhang S, Zhang L, Hao Q: The overexpression and predictive significance of MMP-12 in esophageal squamous cell carcinoma. Pathology-Research and Practice 2017, 213(12):1519-1522.

24. Holmström SB, Lira-Junior R, Zwicker S, Majster M, Gustafsson A, Åkerman S, Klinge B, Svensson M, Boström EA: MMP-12 and S100s in saliva reflect different aspects of periodontal inflammation. Cytokine 2019, 113:155-161.

25. Rahamthulla SU, Priya PV, Hussain SJ, Nasyam FA, Akifuddin S, Srinivas VS: Effectiveness of the supraomohyoid neck dissection in clinically NO neck patients with squamous cell carcinoma of buccal mucosa and gingivobuccal sulcus. Journal of International Society of Preventive \& Community Dentistry 2015, 5(2):131.

26. Mishev G, Deliverska E, Hlushchuk R, Velinov N, Aebersold D, Weinstein F, Djonov V: Prognostic value of matrix metalloproteinases in oral squamous cell carcinoma. Biotechnology \& biotechnological equipment 2014, 28(6):1138-1149.

27. Yen C-Y, Chen C-H, Chang C-H, Tseng H-F, Liu S-Y, Chuang L-Y, Wen C-H, Chang H-W: Matrix metalloproteinases (MMP) 1 and MMP10 but not MMP12 are potential oral cancer markers. Biomarkers 2009, 14(4):244-249.

28. Feng Y, Li Q, Chen J, Yi P, Xu X, Fan Y, Cui B, Yu Y, Li X, Du Y: Salivary protease spectrum biomarkers of oral cancer. International journal of oral science 2019, 11(1):7.

29. Impola U, Uitto V-J, Hietanen J, Hakkinen L, Zhang L, Larjava H, Isaka K, Saarialho-Kere U: Differential expression of matrilysin-1 (MMP-7), 92 kD gelatinase (MMP-9), and metalloelastase (MMP-12) in oral verrucous and squamous cell cancer. The Journal of Pathology: A Journal of the Pathological Society of Great Britain and Ireland 2004, 202(1):14-22.

30. Cai X, Yao Z, Liu G, Cui L, Li H, Huang J: Oral submucous fibrosis: A clinicopathological study of 674 cases in China. Journal of Oral Pathology \& Medicine 2019, 48(4):321-325.

31. Yang S-F, Wang Y-H, Su N-Y, Yu H-C, Wei C-Y, Yu C-H, Chang Y-C: Changes in prevalence of precancerous oral submucous fibrosis from 1996 to 2013 in Taiwan: a nationwide population-based retrospective study. Journal of the Formosan Medical Association 2018, 117(2):147-152.

32. Yang PY, Chen YT, Wang YH, Su NY, Yu HC, Chang YC: Malignant transformation of oral submucous fibrosis in Taiwan: a nationwide population-based retrospective cohort study. Journal of Oral Pathology \& Medicine 2017, 46(10):1040-1045.

33. Speight PM, Khurram SA, Kujan O: Oral potentially malignant disorders: risk of progression to malignancy. Oral surgery, oral medicine, oral pathology and oral radiology 2018, 125(6):612-627.

34. Shaikh AH, Ahmed S, Siddique S, Iqbal N, Hasan SMU, Zaidi SJA, Ali A: Oral submucous fibrosis The Professional Medical Journal 2019, 26(02):275-281. 